\title{
CUIDATS: An RFID-WSN hybrid monitoring system for smart health care environments
}

\author{
Toni Adame ${ }^{\mathrm{a}}$, Albert Bel ${ }^{\mathrm{a}}$, Anna Carreras $^{\mathrm{a}}$, Joan Melià-Seguíb,c ${ }^{\text {, }}$ Miquel \\ Oliver $^{\mathrm{a}}$, Rafael Pous ${ }^{\mathrm{a}}$ \\ ${ }^{a}$ Department of Information and Communication Technologies \\ Universitat Pompeu Fabra \\ Carrer de Roc Boronat 138, 08018 Barcelona - Spain \\ ${ }^{b}$ Estudis d'Informàtica, Multimèdia i Telecomunicació \\ Universitat Oberta de Catalunya \\ Rambla del Poblenou 156, 08018 Barcelona - Spain \\ ${ }^{c}$ Internet Interdisciplinary Institute \\ Universitat Oberta de Catalunya \\ Av. Carl Friedrich Gauss 5, 08860 Castelldefels - Spain
}

\begin{abstract}
The continuous development and improvement of low-power wireless communication technologies is enabling the emergence of many new applications in the field of the Internet of Things (IoT). One of the main areas of research within the Smart City context is smart health, which engages novel IoT initiatives to improve both quality and access to health care and smart services in general. In this paper we present CUIDATS, an IoT hybrid monitoring system for health care environments which integrates RFID and WSN technologies in a single platform providing location, status, and tracking of patients and assets. After its performance validation in a suitable testbed, CUIDATS has been deployed and evaluated with a high degree of success in a real hospital.
\end{abstract}

Keywords: Smart Health, RFID, WSN, Internet of Things, RTLS

Email addresses: toni.adame@upf .edu (Toni Adame), albert.bel@upf .edu (Albert Bel), anna.carrerasc@upf .edu (Anna Carreras), melia@uoc.edu (Joan Melià-Seguí), miquel.oliver@upf.edu (Miquel Oliver), rafael.pous@upf .edu (Rafael Pous) 


\section{Introduction}

Urban population has been growing for the last decades and it is expected to continue growing in the future, especially in less developed countries. The management of urban areas has been identified by the United Nations as one of the most important development challenges of the 21st century [1]. In this context, a strong effort on research is being conducted under the umbrella of the Smart City concept. The main objective is to design and deploy sustainable cities, not only from an environmental point of view, but also from technological, industrial, management, and social perspectives. To achieve the above goals, smart systems together with smart processes are increasingly being used. For instance, increasing efforts are also being conducted in the context of smart health care nowadays [2]. This is a strategical societal challenge due to two main reasons: on the one hand, the world's population aging is forcing rapid rises in health care costs, and on the other hand, the current economic recession has brought many governments to reduce public health budgets. Improving the efficiency of health care infrastructures and medical systems while reducing their costs is, thus, a primary issue towards achieving smart health care.

For instance, automatizing common medical processes such as identification, localization, and monitoring of patients and assets, which are still being done manually nowadays, is a requirement in smart health care designs. In this context, Internet of Things (IoT) is the main front-end for the Smart City technologies. A diverse and interconnected network of sensors and actuators is the suitable candidate to develop innovative smart systems. More precisely, the integration of Radio Frequency Identification (RFID) and Wireless Sensor Networks (WSNs) guarantees a low-cost and extensible infrastructure for the identification and tracking of both users and assets. The integrated RFID/WSN network provides a centralized and autonomous performance, that is, without requiring any human intervention.

On the one hand, RFID technology provides an unassisted and automated identification system at object level. Specifically, the Ultra High Frequency (UHF) Electronic Product Code Class 1 Generation 2 (EPC Gen2 for short) [3] provides an identification read range of about six meters using passive tags. These tags can also incorporate sensors (i.e. temperature or accelerometer), although depending on the configuration, the read range may be decreased. On the other hand, WSN technology leverages its autonomous, scattered, ubiquitous, low-powered, and non-invasive nature for 
acquiring and cooperatively transmitting data within a self-configurable network.

In this paper we summarize the main outputs of the 2-year project CUIDATS (meaning "cared-for" in Catalan language), formed by Proengsoft 1 , Pulso Ediciones ${ }^{2}$, i2cat ${ }^{3}$, and UPF ${ }^{4}$. At its first stage, the project identified two main drawbacks in existing smart health solutions: first, the lack of integration between different IoT technologies; and second, the lack of implementations in real scenarios including integration with the Hospital Information System (HIS). Next, different RFID and WSNs integration scenarios were analyzed, selecting the best approach based on state-of-the-art technologies.

With this acquired knowledge and the valuable involvement of a medical institution, the project consortium designed, built and evaluated a platform for the automatic identification, localization, and monitoring of patients and assets, whose main contributions are detailed below:

- Design and implementation of a hybrid network consisting of RFID tags and readers, WSN beacons, and a gateway acting as a common element between both wireless technologies.

- Development of an RTLS system running over the hybrid network to accurately locate patients and assets.

- Development of an electronic wristband to track patients and monitor their vital signs within a health care environment.

- Testing and pilot execution in a real health care scenario.

The remainder of this paper is organized as follows: Section 2 highlights existing works related to the use of RFID and WSNs in health care as well as in other application scenarios. Section 3 presents the overall CUIDATS platform while Section 4 goes into details of the used technologies and their integration. Section 5 describes and evaluates the pilot deployed in a real health care institution, and finally, Section 6 concludes the paper and identifies future work.

\footnotetext{
${ }^{1}$ http://www.proengsoft.com/

${ }^{2}$ http://www.pulso.com/

${ }^{3}$ http://www.i2cat.net/

${ }^{4}$ http ://www .upf .edu/
} 


\section{State of the Art}

In health care, as in many other application fields, any automatic system responsible for identifying, locating, and tracking objects or people within a pre-defined area is encompassed within the RTLS concept.

By helping companies to locate their patients and assets effectively and efficiently, RTLS solutions deliver simpler operations, increased asset utilization, reduced hospitalization time, lower number of complaints, and enhanced productivity, resulting in tangible benefits and accelerated return of investment (ROI) [4].

Over the last years, the use of RTLS for patient tracking and asset management in health care environments has experienced a sustained growth. However, it is expected a prominent adoption of this kind of systems from 2014 to 2020 , with a compound annual growth rate of $32.9 \%$ and a estimated world-wide market of around $\$ 2,000$ million by 2020 [5].

\subsection{Technologies and features}

There currently exists a wide range of communication technologies that can be used alone or in combination with others to form an RTLS. Most solutions are based on RF technologies, whose operating principles for indoor location do not differ significantly from the ones used by GPS in outdoors. Thus, these systems are able to estimate the position of a transmitter from the analysis and comparison of its emitted wavelength, including $W i F i$, RFID, Bluetooth, Bluetooth Low Energy (BLE), Ultra-wideband (UWB), Zig$B e e$, and other proprietary solutions.

And yet there is also room for optical and acoustic technologies, both having in common its inability to penetrate walls, since only tags and receivers that have line of sight can communicate. Examples of these technologies are Infrared (IR) and Second Generation Infrared (Gen2IR), and Ultrasound, respectively. Regardless the employed technology, any RTLS architecture consists of the following elements:

Tag/Badge This element is responsible for transmitting the identifier (ID) and other possible information, if any, gathered from the patient/asset to which is attached in a periodic manner (active mode) or after having been interrogated by a location sensor (passive mode).

Location sensors These receivers are placed throughout the health care facility and gather all the information transmitted by tags. They also 
may perform some processing before retransmitting data to the location engine.

Location engine It is probably the most important element of the system, as it contains all the computational power to precisely estimate the position of people and objects in real time.

Middleware It can be described as the software connector between the pure RTLS technology elements (tags, sensors, and the location engine) and the business applications, by translating raw data into usable information.

Applications These computer programs interact with the RTLS middleware to boost the efficiency of health care processes. Besides, they can be integrated into the HIS for a greater data availability and usability.

Depending on the kind of technology and the density of deployed sensors, an RTLS may contain different accuracy levels: Zone-level, where the location of an item is given on the correct floor or within a pre-determined area (Building 2, Floor 4; or admissions area); Room-level, which locates a person or asset in an specific room (patient room 320, or Operating Room 4); and Bed-level, where a person or asset is located in a specific sub-location within a room (patient room 320, Bed 1 or ED exam 2).

Lastly, to achieve a certain level of accuracy in the positioning system, a RTLS may use geometric (trilateration or triangulation) or statistical techniques fed by one or several of the following signal parameters: received signal strength (RSS), angle of arrival (AOA), time of arrival (TOA), and time difference of arrival (TDOA) [6].

\subsection{Related Research}

Among the research community, we have taken into consideration two kinds of RTLS initiatives: those which combine RFID and WSN in any application field and those applied directly on health care environments. In the first group, four different types of integration between RFID and WSN technologies according to [7] are presented:

1. Integration of RFID Tags with Sensors These tags are capable of acquiring sensor data and using RFID-specific protocols for transmitting both the RFID frame and the sensor data [8]. Depending on its energy source, RFID technology is classified as: 
(a) Active RFID

RFID circuitry, communications, and sensors are powered by a battery, thus extending the features of an RFID network with sensing capabilities [9-11].

(b) Semi-passive RFID

Extending the reading range by using a WSN and prolonging the battery life of semi-passive RFID tags (which use a battery for their circuits, but need power coming from the reader to communicate) are the main goals in [12].

(c) Passive RFID

These tags do not contain a battery and rely entirely on the reader as their power source. They may include a handful of different sensors [13 16], provided that they can also be activated by the RFID reader.

2. Integration of RFID Tags with WSN Nodes

Conversely, WSN nodes are in this case equipped with RFID tags and incorporate an identification code to their readings. There exist solutions using different WSN technologies, such as IEEE 802.15.4 [17], ZigBee [18, and even WiFi or Bluetooth [19].

3. Integration of RFID Readers with WSN Nodes and Wireless Devices This integration solution implies the connection of an $\mathrm{RF}$ radio module to an RFID reader. Different approaches are proposed in [10, 20 23].

4. Middleware-Level Integration of RFID Devices with WSN Nodes Performed in the middleware-layer, this kind of integration maintains the physical separation between both technologies, as shown in 24 26].

In the second group, we find different surveys of RFID [27], WSN [28] and integrated RFID-WSN [29] use cases for building a smart hospital. As for real implementations, [30] uses RFID to detect interactions in ambient assisted living environments in order to improve lives of people with physical or cognitive disabilities; an RFID-WSN mote is used for monitoring patients' medication in [31] and physical parameters in [32. Transfers in beds, chairs and walking are monitored with passive RFID in [33; an hybrid RFIDWSN system for blood bags management is proposed in [34]; and a ZigBee wristband with measurement of pulse, skin conductivity, and momentum is presented in [35]. 


\subsection{Wearable sensors in eHealth}

In recent years, extensive efforts have been made in the research and development of smart wearable sensors for health monitoring, due primarily to recent technological advances in micro- and nanotechnologies, miniaturisation of sensors, and smart fabrics [36]. This plethora of sensors can be classified into contact and non-contact ones, with characteristic examples of the analyzed biosignals shown in Table 1 .

1. Contact sensors: They consist of a recognition element (receptor) coupled with a transduction element. In the receptor part, the biochemical or electrical/mechanical information is converted into a form of energy that can be measured by a transducer.

2. Non-contact sensors: The variable under study is analyzed after generating an optical, radiofrequency or ultrasonic signal and evaluating the response received back by the sensor. In the case of inertial sensors, they rely on their accelerometers, gyroscopes and even magnetometers to obtain motion data.

\begin{tabular}{|l|l|l|}
\hline Type & Subtype & Biosignals \\
\hline \multirow{3}{*}{$\begin{array}{l}\text { Contact } \\
\text { sensors }\end{array}$} & Biochemical & $\begin{array}{l}\text { Body temperature, blood glucose level, } \\
\text { galvanic skin conductance, sweat } \\
\text { analysis (pH level and profile of chemicals) }\end{array}$ \\
\cline { 2 - 3 } & Mechanical/Electrical & Respiratory rate, ECG, EMG, EEG \\
\hline \multirow{2}{*}{$\begin{array}{l}\text { Non-contact } \\
\text { sensors }\end{array}$} & Ultrasonic & Blood pressure \\
\cline { 2 - 3 } & Radiofrequency & $\begin{array}{l}\text { Respiratory rate, blood glucose level, } \\
\text { heart rate and rhythm, location }\end{array}$ \\
\cline { 2 - 3 } & Optical & $\begin{array}{l}\text { Body temperature, heart rate and rhythm, } \\
\text { blood oxygen saturation }\end{array}$ \\
\cline { 2 - 3 } & Inertial & $\begin{array}{l}\text { Fall detection, walking distance and speed, } \\
\text { step count, stride distance and length, } \\
\text { body posture }\end{array}$ \\
\hline
\end{tabular}

Table 1: Main biosignals measured by wearable sensors from eHealth applications (based on [37-45])

\subsection{Market players}

Table 2 details the features of the main RTLS market players in the commercial ecosystem. Although there exists a wide range of different technological solutions, none of those systems has prevailed because of the current diversity of application environments and specific technological problems. 
WiFi- and ZigBee-based solutions [46 52] share the $2.4 \mathrm{GHz}$ channel spectrum with the 802.11 protocol, producing interference issues with some other wireless applications. Besides, their lack of accuracy (averaging as much as 10 meters) makes them not suitable for locating systems. Lastly, while WiFi-based RTLS can leverage existing infrastructures (at the cost of adding complexity and security issues to data transmission), ZigBee-based solutions need to include a high volume of plug-in sensors everywhere due to the low coverage range of their radio modules.

With regard to ultrasound and IR solutions [53 55], they require a clear line of sight between tags and sensors to communicate, producing momentary losses of patient track if a tag is hidden, for instance, by a blanket. Furthermore, these technologies require a dense deployment of receivers and dedicated wiring infrastructure, thus making facility-wide RTLS adoption cost-prohibitive.

RFID systems such as [56] take advantage from its low cost and versatility, but only provide tagged object's location when a reader is nearby (e.g., 3 meters). They also fail when trying to transmit efficiently all the gathered information to a central server, usually relying on another communication system to provide real-time monitoring. 


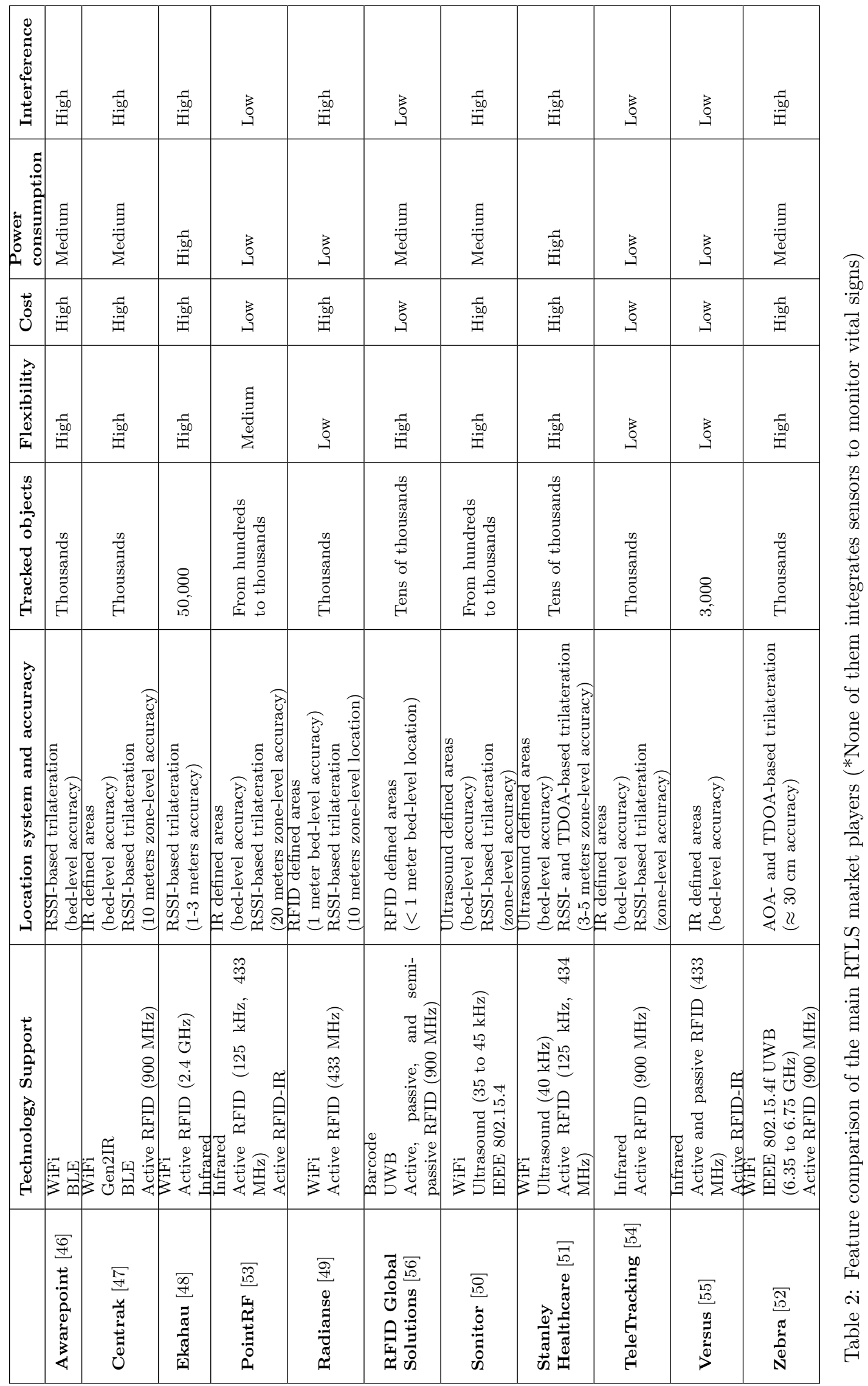




\subsection{Motivation}

The motivation of the CUIDATS project arises from the lack of any academic or commercial RTLS for healthcare able to properly integrate the acquisition of biosignal data from patients, assets inventory and localization, and its transmission to a central server by means of wireless technologies with the following requirements (see Table 2):

- Flexible: easily integrable with different standard communication protocols and existing devices, biosignal sensors, and hospital information systems. Moreover, RFID readers can read hundreds of tags simultaneously, allowing any number of asset tracking in practice.

- Non-invasive: no break in the skin is created and there is no contact with the mucosa during the process of acquiring biosignal data.

- Low-cost: wristband based on proven elements with low cost of production, and asset monitoring based on battery-less passive RFID tags.

- Low-powered: WSN and wristband are composed of integrated mechanisms to reduce power consumption and enlarge device's battery lifetime. In addition, passive RFID tags do not require battery since are powered by the RFID reader.

- Minimally interfering: without affecting the hospital's daily routines nor obstructing the proper operation of other wireless systems. The proposed system can be deployed without requiring any modification in the hospital data network infrastructure, since the WSN is able to manage all the information generated from the sensors.

\section{CUIDATS Platform}

CUIDATS is a monitoring solution for tracking patients and assets in a health care environment. Besides, it also monitors patients' vital signs (temperature, pulse, and fall alert) and activates alarms depending on preestablished thresholds. Conceived as a supplementary system to certified medical devices, CUIDATS provides $24 / 7$ control of patients inside a health care environment.

While assets can be tagged with active or passive RFID tags, patients wear a wristband equipped with different vital sign sensors and an RF trans-

mitter. In turn, RFID readers and RF beacons are deployed throughout the 


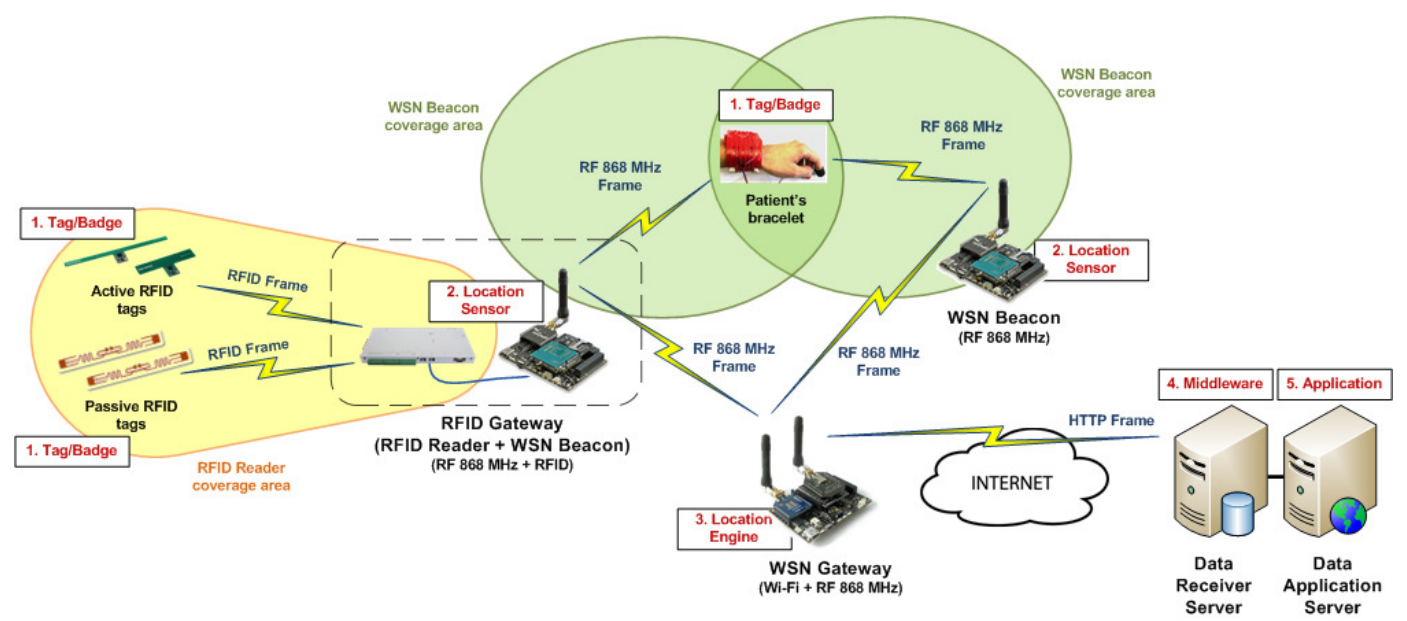

Figure 1: Architecture of CUIDATS hybrid monitoring system

health care facility, so that they gather all the information from assets' tags and patients' wristbands.

One of the most striking features of CUIDATS is that RFID readers are fully integrated with RF beacons in a single device, thus creating a WSN mesh responsible for retransmitting the gathered data to the CUIDATS server. In addition, the whole system may be easily integrated with the HIS, so that the complexity of the mesh network is alleviated whereas the general usability of the platform is increased.

Next, we detail the hardware technologies of the CUIDATS monitoring platform (see Figure 1), as well as their integration into a single hybrid network.

\subsection{RFID}

Within the different RFID technologies and standards, the passive UHF EPC Gen2 [3] has been used in this project. It defines at the link layer the identification protocol and the necessary messages between reader and tags for a successful identification. Next, we enumerate the main reasons that led us to choose this standard, in line with the goals of the CUIDATS project. First, its largest reading range (about six meters) within the passive technologies makes it suitable to detect RFID tags, for instance, when passing through doors. It is also a world-wide standard, offering full compatibility regardless of the tags origin. Moreover, the EPC Gen2 tags are designed to balance cost and functionality, turning their cost to a few cents 
of Euro/Dollar, and thus, allowing the labeling of any individual object at a very low cost. Finally, it is a passive technology, meaning that the tags are battery-less, easing the patient and asset monitoring.

The EPC Gen2 standard allows the extension of the identification protocol to custom commands. This is used by different manufacturers to extend the functionality of the system, for instance, to add sensors on board EPC Gen2 tags. These sensors may be powered with batteries (semi-passive technology) or passively, in which case the read range is typically reduced to allow enough energy for the sensors to power-up and run [57].

We propose the utilization of the EPC Gen2 RFID technology to monitor both assets (using standard tags) and patients (using tags provided with sensors). The following devices are used in the implementation of the system:

Standard tags ShortDipole Impinj Monza 5 tags [58] were chosen due to its correct performance close to metallic items, which is a common requirement for health care assets. These tags provide the standard 96bit identification, at an average range of 400 identified tags per second. In our scenario we expect a few tens of objects to be identified within a second at most (a trolley with different assets passing through a door).

Tags with sensors Based on the requirements of the project, we tested accelerometer's (to detect patient falls) and skin temperature. The Kineo [59] and Pyros [60] tags from the company Farsens were used in the implementation of the project. These tags provide the common 96-bit identification, before performing a number of Access queries to request the sensor data (cf. Table 3). Due to these additional steps in the process, only a few tags per second can be fully accessed. Nevertheless, it is an assumable requirement in our scenario since we only consider one person passing through a door at a time.

Reader Any EPC Gen2 compatible reader could be used for the purposes of the above tasks. However, we required additional features for a successful integration with the CUIDATS platform. On the one hand, we needed the reader firmware to be configurable to allow enough time for the tags with sensors to be powered-up [57]. On the other hand, we needed the reader to have an available communication port to exchange data with the WSN system. The Keonn AdvanReader-150 [61] was chosen for the integration, since it fulfilled the above requirements. 


\begin{tabular}{|l|l|l|l|}
\hline Tag & Identification & EPC & Information \\
\hline Impinj Monza 5 & Assets & $0 *_{\mathrm{b}}$ & 96 bits $($ EPC $)$ \\
\hline Farsens Kineo & Patient orientation & $1 *_{\mathrm{b}}$ & $96+48$ bits $($ EPC + XYZ orientation $)$ \\
\hline Farsens Pyros & Patient temperature & $1 *_{\mathrm{b}}$ & $96+16$ bits $($ EPC + temperature $)$ \\
\hline
\end{tabular}

Table 3: Information included in RFID tags.

Since the EPC Gen2 RFID only defines its performance up to the link layer, an additional technology carrying the RFID messages on the upper network/transport layers is necessary. The next subsection details the performance of the WSN, which handles this task, besides its own performance as a sensing network.

\section{2. $W S N$}

The CUIDATS WSN architecture is based on the model offered by Waspmote $868 \mathrm{MHz}$ devices [62], consisting of four distinguished layers:

Physical Layer Among the different available radio modules for the Waspmote platform, the Digi XBee-PRO 868 was selected due to its wide signal coverage and low signal attenuation in indoor environments. In addition, the availability of up to 5 different transmission modes ( 0 , 13.7, 20, 22, and $25 \mathrm{dBm}$ ) make this device suitable to incorporate battery- and link-aware energy saving mechanisms.

MAC Layer XBee-PRO 868 MAC layer is a proprietary development that provides point-to-point, reliable communications by using retries and acknowledgments. Data integrity is ensured by appending a cyclical redundancy check (CRC) code at the end of each outgoing RF packet. Each radio module is identified by a unique 64-bit MAC address and can perform unicast and broadcast communications with payloads of up to 100 bytes.

Network Layer As XBee-PRO 868 devices do not include any network layer by default, it has been necessary to implement a new one from scratch. CUIDATS network layer includes all the logic to deploy a mesh network, so that WSN beacons reconfigure themselves according to network conditions, thus creating connections resistant to disaster and other interference. The main element of this new network layer is a routing protocol which uses as decision metrics the number of hops to 
the WSN gateway and the received signal strength indication (RSSI). Thus, each WSN beacon initially tries to establish a single-hop connection with the WSN gateway. If it were not possible, the search for an alternative route would be activated.

Security Layer The security layer encrypts any outgoing data (for RF transmission) using AES-128 bit scheme. Similarly, when RF data packets are received (and encrypted), the security layer is responsible for decrypting them.

Due to the scarce battery lifetime of the employed devices, several mechanisms have been designed in order to ensure minimum energy consumption, such as: a handshake between transmitter and receiver which selects the most appropriate power transmission mode according to the distance between both devices and their corresponding battery levels, an automatic routine for deactivating the radio module during non-traffic periods, and a low-battery detection and notification alarm system.

\subsection{CUIDATS Wristband}

A customized wristband (Figure 2) was built by using 3D printing technology at the Fab Lab Barcelona fabrication laboratory 5. The following requirements were taken into account during the design stage:

- Inclusion of three vital signs sensors: temperature, pulse, and fall alert

- Reduced dimensions device (similar to a wristwatch)

- Capacity to contain all elements of the system: transmitter radio module, battery, sensors, and circuitry

- Ergonomic and flexible solution

- Suitable to be washed and/or sanitized by the health center

- Easy to power on and off

- Easy battery removal for recharging and/or replacement

\footnotetext{
${ }^{5}$ http://www.fablabbcn.org
} 


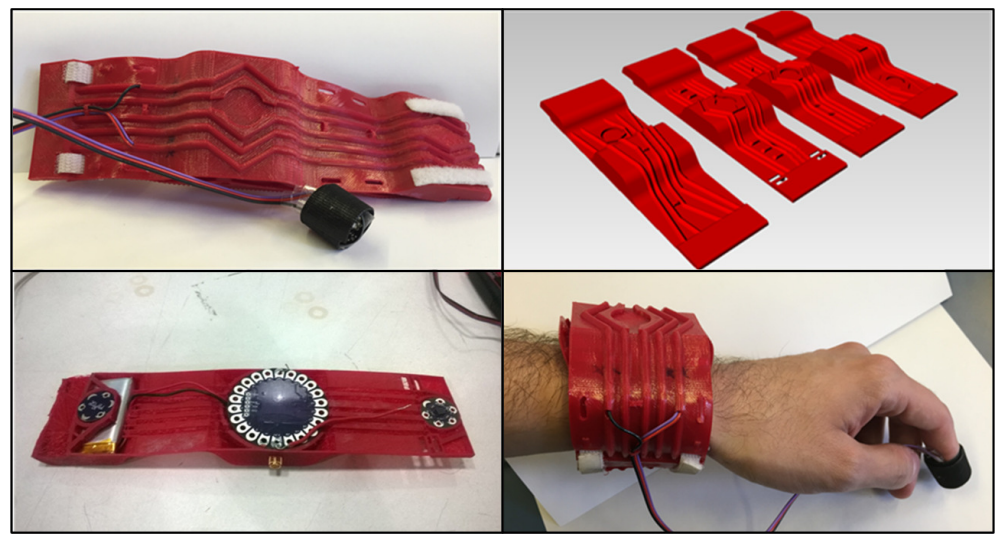

Figure 2: CUIDATS Wristband

\begin{tabular}{|l|l|}
\hline Function & Element \\
\hline Casing & PLA Plastic \\
\hline Board & LilyPad XBee \\
\hline Radio module & Digi XBee 868 MHz \\
\hline Battery Board & LilyPad LiPower \\
\hline Battery Charger & SparkFun LiPo Charger Basic (Micro-USB) \\
\hline Battery & Polymer Lithium Ion Battery (1000 mAh) \\
\hline Temperature Sensor & LilyPad MCP9700 \\
\hline Pulse Sensor & Pulse Sensor Amped \\
\hline Accelerometer & LilyPad ADXL335 \\
\hline
\end{tabular}

Table 4: List of elements of the CUIDATS wristband

The wristband was designed to acquire accurate information about the temperature, pulse, position and movement of the patient. Gathered data is later used to reproduce the physiological signals of the patient as well as to activate an alarm if any pre-established threshold is surpassed. Temperature and pulse alarms are set off if analyzed values are out of the ordinary, whereas brusque movements detected by the accelerometer are considered as falls and reported to the sanitary personnel. In addition, the position of the patient is estimated through a localization and tracking algorithm which can be enriched in combination with the web platform by applying different rules to prevent possible escapes or access to private areas.

The final model of the wristband was validated after successive prototype iterations including all the technological elements from Table 4 and once the desired performance was achieved. 


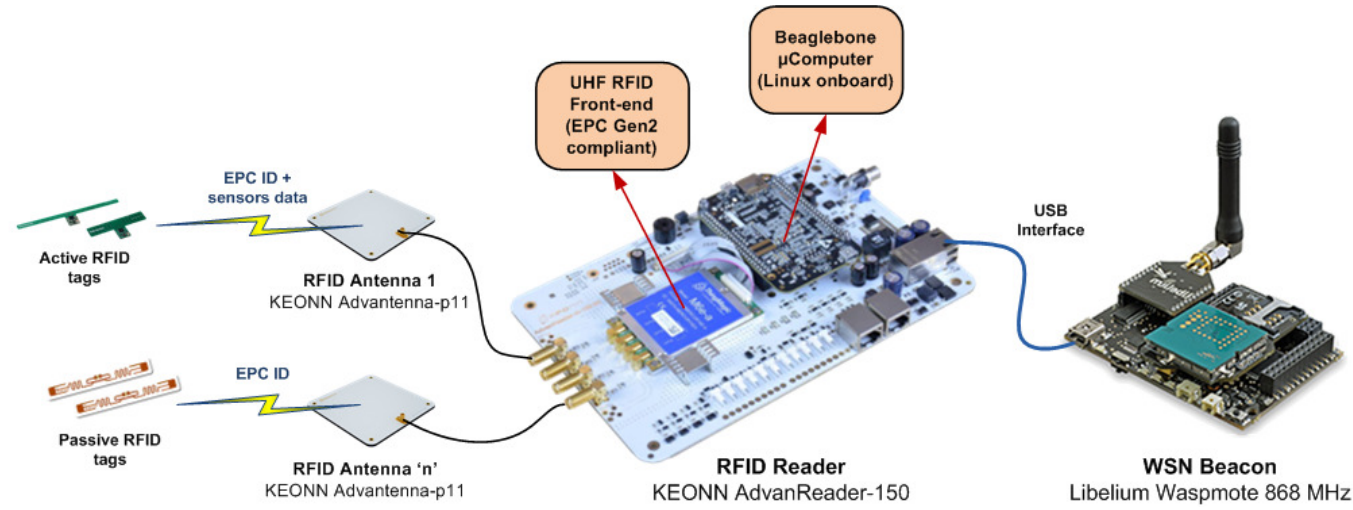

Figure 3: RFID Gateway architecture

\subsection{RFID-WSN Integration}

One of the major innovations introduced by the CUIDATS project is the complete integration of RFID readers with the WSN. Thus, a compact, easily transportable device called RFID Gateway has been designed and built, whose main task is to identify people and assets within its coverage area and report the obtained data to the WSN. As shown in Figure 3, it consists of four main elements:

Antennas RFID antennas have a double functionality. On the one hand, they generate an electromagnetic field which powers the passive RFID tags (and, if applicable, sensors). On the other hand, they receive data from those tags. In the current project, several Keonn Advantenna-P11 [63] have been used, with $100^{\circ}$ directivity and $3.2 \mathrm{dBi}$ gain.

UHF RFID Front-end The RFID Front-end is the device which transforms binary digital information to $\mathrm{RF}$ signals ready to be transmitted. In order to handle the tags diversity, the Keonn AdvanReader-150 (detailed in Section 3.1) identifies tags regarding their EPC identifier (cf. Table 3). If the most significant bit is codified to zero, the reader identifies the tag following the standard EPC Gen2 procedure. If the most significant bit is codified to one, meaning the tag has sensors on board, the reader enters in the Access stage after the actual identification to retrieve the sensor's information [3, 59, 60].

Microcomputer A microcomputer (Beaglebone Black [64]) is responsible for managing the RFID front-end operation and preprocessing the received 
information. It runs a Python application over a Debian 7 Linux distribution, which performs a query-response protocol to reliably transmit the RFID data to the WSN beacon over a serial port, hence, ensuring the correct transmission of all RFID messages. Specifically, the Python application constantly requests the RFID reader whether new tags have been identified. If affirmative, the information is sent to the WSN beacon requesting acknowledgment of the proper reception. In case of negative acknowledgment or timeout, the message is sent again. Lastly, RFID readings along with information resulting from wristbands are transmitted from the WSN beacon through the WSN.

WSN Beacon It is connected to the microcomputer via an USB cable and is responsible for transmitting the gathered information from RFID tags to the WSN. This Waspmote $868 \mathrm{MHz}$ beacon [62] is equal to the others used in the CUIDATS WSN, and may also receive, process and retransmit data from wristbands.

Battery (optional) Although the RFID Gateway is by default powered through power line, it can also work on batteries if required.

The proposed RFID Gateway contributes the following features, in line with the CUIDATS platform goals:

- The RFID Gateway improves the efficiency of the system by allowing the unified monitoring of both assets and patients. On the one hand the assets are tracked with passive RFID labels. On the other hand, the patients can also be monitored using passive RFID tags with sensors (cf. Table 3).

- The degree of information knowledge within the hospital is augmented thanks to a real-time positioning of the assets, as well as patients. For instance, if the patient is close to an RFID Gateway, their information can be monitored through the passive RFID tag with sensor, and thus, reducing battery consumption from the wristband. Moreover, if further information is required (i.e. additional tracking points), new RFID Gateways can automatically be integrated into the current platform.

- All RFID monitoring is transparent to the WSN network (RFID data is integrated in WSN packets at the gateway), adding simplicity to the platform management. Moreover, the RFID Gateway improves the 


\begin{tabular}{|l|l|}
\hline Frontend & Apache 2.4 \\
\hline Web server & PHP and HTML5 \\
\hline Frameworks & jQuery and jQuery UI \\
\hline JavaScript libraries & Bootstrap CDN \\
\hline Content delivery network & \\
\hline Backend & Java \\
\hline Core application & MySQL \\
\hline Database &
\end{tabular}

Table 5: List of CUIDATS web platform technologies

cost-effectiveness of the system since each asset can be tracked with a RFID tag whose cost is a few cents of Dollar/Euro. The batteryless passive RFID tags used for inventorying also add simplicity to the system since they will never require a battery replacement.

\subsection{Communication with the data server}

The communication with the data receiver server is performed through a link between the WSN gateway and a WiFi access point (AP). The existing wireless infrastructure of the hospital has been leveraged to create a private and dedicated VLAN for this purpose.

Communications security is ensured by WPA-Personal protocol, where each wireless network device encrypts traffic using a 256-bit key. Data transmission is based on the client-server model over the HTTP protocol and messages are encapsulated following the JSON data format. In addition, and for synchronizing the whole network, the WSN gateway periodically takes from the HTTP server a timestamp which is properly spread to the rest of WSN Beacons.

\subsection{Web platform}

The web platform, whose main technologies are detailed in Table 5, was entirely developed by Proengsoft and is responsible for receiving the data from the WSN gateway, storing it in a database and showing it in an userfriendly interface. The position of users and assets is easily recognizable on the different hospital floor plants and further information is obtained by simply clicking on them. In addition, the platform also manages the incoming alarms (such as a patient escape or a sudden worsening of a patient's health) and initiates the pre-established protocols. 
Several platform modules let hospital staff configure most of system parameters depending on their profile and authorization, such as registering new users or assets, establishing alarm thresholds, defining hospital areas, or modifying alarm protocols.

\section{Testbed}

In this section we present an evaluation of the CUIDATS platform through a testbed including all the proposed features for smart health monitoring.

\subsection{Evaluation of functionalities}

Next, smart health-related features such as RTSL, fall alert detection, and vital signs monitoring are evaluated.

\subsubsection{Real-Time Locating System (RTLS)}

In order to track the patients wearing the wristband, we decided to use a weighted centroid algorithm. This localization algorithm offers a simple solution to obtain the patient's position. The WSN gateway has the responsibility of collecting different packets from the entire network. These packets, sent by WSN beacons, include the collected RSSI values from wristband

messages. Hence, as we centralize the position estimation at the gateway, a low-complex algorithm is used.

The weighted centroid algorithm is a range-based method with a high dependency on the number of reference nodes $(n)$ and their distribution throughout the scenario [65]. The reliability of this algorithm may be increased by introducing a weight $\left(w_{i}\right)$ [66], which gives more importance to those nodes very close to the non-positioned node. Hence, the position $(x, y)$ of the wristband is obtained by using Equation 1 ;

$$
(x, y)=\left(\frac{\sum_{i=1}^{n} w_{i} x_{i}}{\sum_{i=1}^{n} w_{i}}, \frac{\sum_{i=1}^{n} w_{i} y_{i}}{\sum_{i=1}^{n} w_{i}}\right),
$$

with the weights obtained in Equation 2,

$$
w_{i}=\frac{1}{d_{i}} .
$$

In our case, the distance $\left(d_{i}\right)$ between every reference node and the patient wristband is estimated. This estimation is done assuming a log-normal 


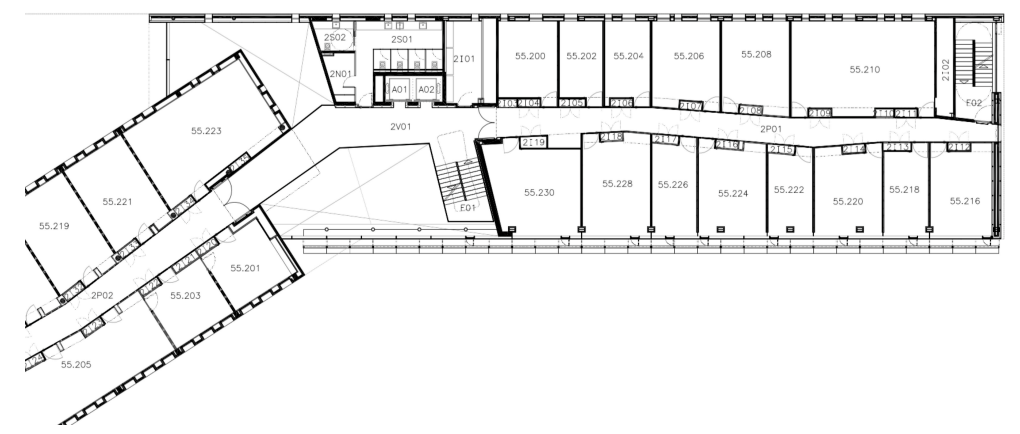

Figure 4: RTLS testbed scenario: UPF facilities

distribution as described in Equation 3, where $P_{0}$ is the received power at a reference distance of 1 meter, $P_{i}$ is the received power by every reference node, and $\alpha$ is the path-loss exponent. It is worth noting that $P_{0}$ and $\alpha$ are obtained from a previous environment characterization.

$$
d_{i}=10^{\frac{P_{0}-P_{i}}{10 \alpha}},
$$

In order to check the feasibility of this localization algorithm previous tests were done at the UPF facilities (see the test scenario in Figure 4). As it is an office building similar to a hospital environment, the achieved results helped us to obtain a performance reference of the selected localization algorithm.

The characterization of the environment was performed through a measurement campaign. Different RSSI measurements at different distances were taken so as to obtain $P_{0}$ and $\alpha$ values, necessary for subsequently estimating distances between nodes. Thanks to the robustdemo function of MATLAB, both parameters were estimated, as can be seen in Figure 5 .

The performance of the localization algorithm is shown in Figure 6. One can observe that the error achieved oscillates between centimeters and some meters, with a mean error of around 5 meters (i.e., zone-level accuracy). This result is accurate enough for the patient tracking application in a hospital and validates the use of the weighted centroid algorithm.

It was also derived from this test that the location of the reference nodes has a huge influence on the results, so that a better distribution of them would enhance the accuracy of the algorithm. Lastly, in order to avoid showing wrong positions (i.e. positions outside the building) we have introduced limits to our estimated positions. Hence, if an estimation is outside the area 


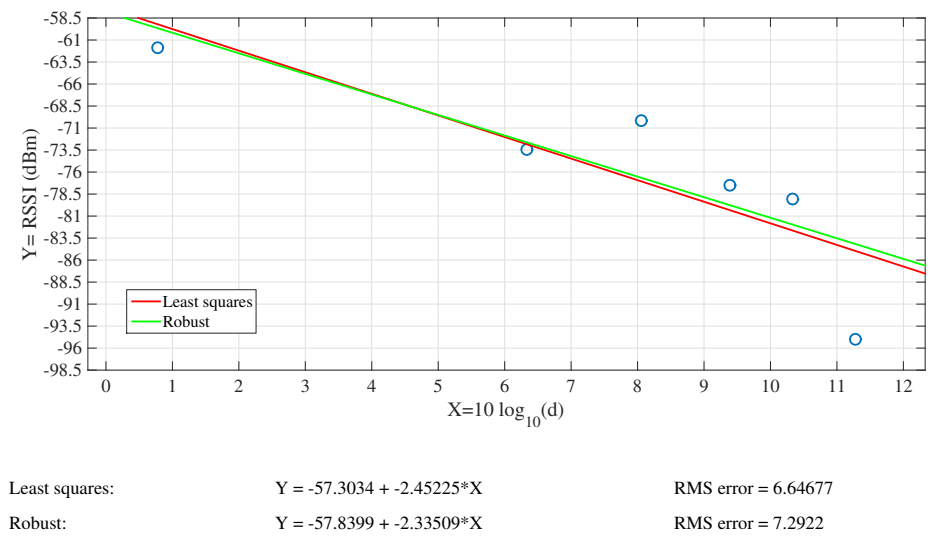

Figure 5: Power characterization of the UPF testbed scenario

of the building, it is relocated to the nearest place inside the considered area.

\subsubsection{Fall alert}

The CUIDATS wristband includes a 3D accelerometer able to detect joint movement as well as inclination and vibration. The WSN beacons read the messages from the wristband and compare the current accelerometer readings, and two previous readings. If this comparison trespasses a certain threshold, the node activates the patient fall alarm and immediately sends a message to the gateway.

The alarm mechanism has been tested in three different cases:

- A brusque fall of the patient's arm when seated (fainting)

- A fall when the patient is quiet

- A fall when the patient is moving

The achieved results are shown in Figure 7. The percentage of correct fall detection is around $70 \%$. We can observe that the worst results are obtained in the walking scenario (Figure 7). It is explained because the movements of the arm when the patient is walking produce false detections.

\subsubsection{Vital signs monitoring}

Another important point of the CUIDATS project is the constant measurement of patients' vital signs. The designed wristband contains temperature and pulse sensors able to provide patient real-time data. 


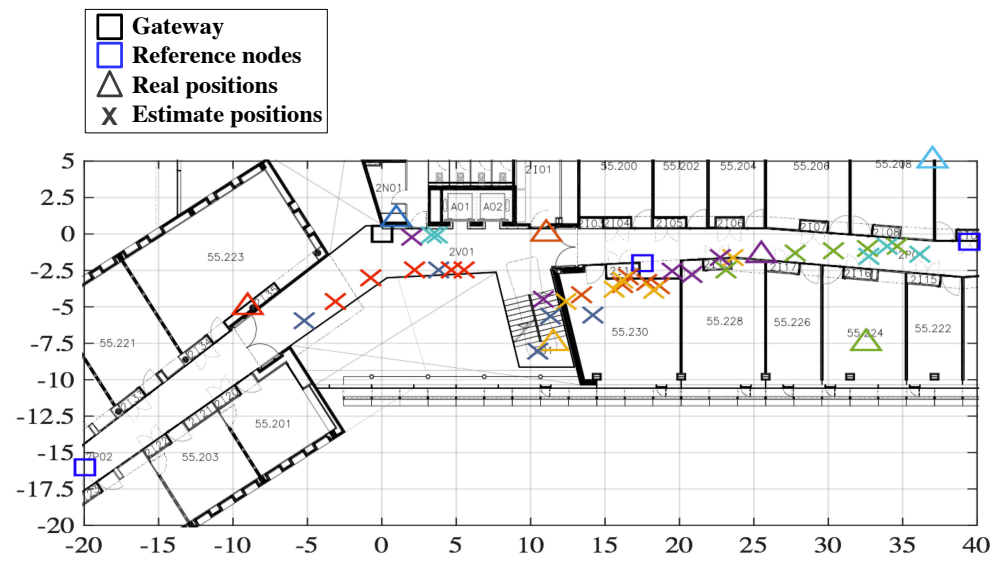

Figure 6: Localization results over UPF floor plan

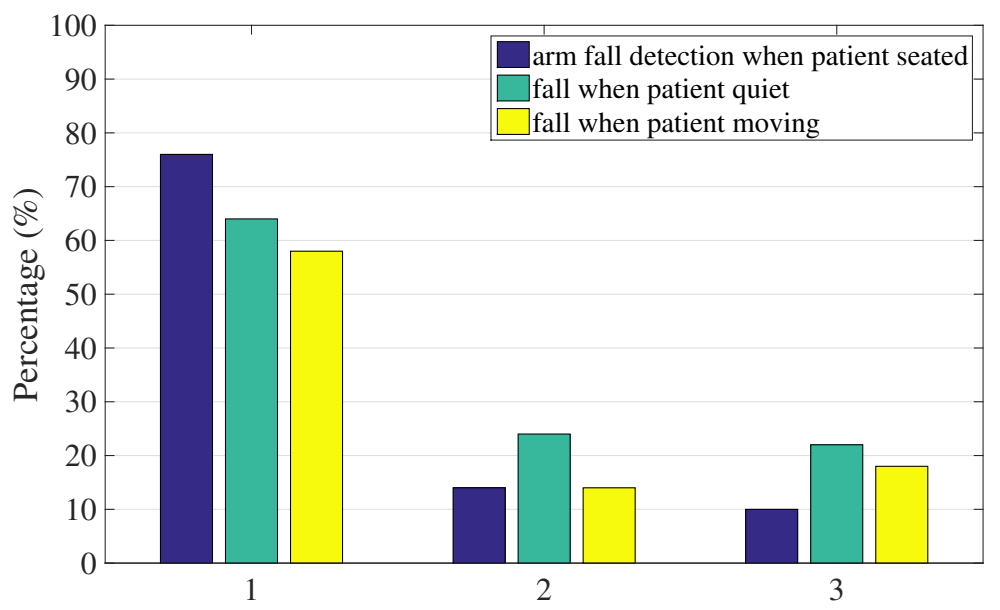

1: Fall detected 2: False fall detected 3: Fall not detected

Figure 7: Fall detection performance 


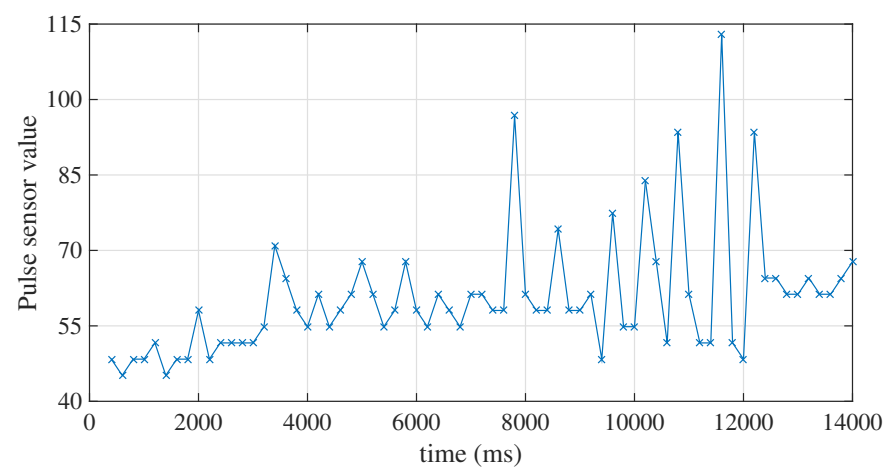

Figure 8: Example of values received from the pulse sensor

The frequency of monitoring messages from the wristband determines its battery lifetime. In that sense, the pulse sensor imposes more restrictions. To obtain a BPM (Beats per Minute) value, the wristband should send a minimum of 35 messages to a node with a maximum inter-message time of $200 \mathrm{~ms}$. In turn, WSN beacons receive these messages, store them and compute the BPM value after detecting the measurement peaks (see Figure 8).

Basically, the computation of the pulse value consists of finding two successive moments of instantaneous heart beat and measure the time between them, called the Inter Beat Interval (IBI). The BPM is derived every beat from an average of the previous 10 IBI times. Some screen captures of our pulse sensor test are shown in Figure 9 .

\subsection{Energy consumption analysis}

To obtain a wristband's battery lifetime estimation, we carried out a real test in a worst case scenario; i.e. the wristband transmitting messages every $200 \mathrm{~ms}$, at the maximum transmitting power, and without any sleeping period. The results show that the battery was active for 6 hours and 30 minutes. Although this lifetime may be suitable for some use cases, we propose in the following measures to increase it:

- A BPM computation every 7 seconds may be too exhaustive. Instead, we propose to compute measurements less often and establish sleeping periods once a BPM value has been computed. 


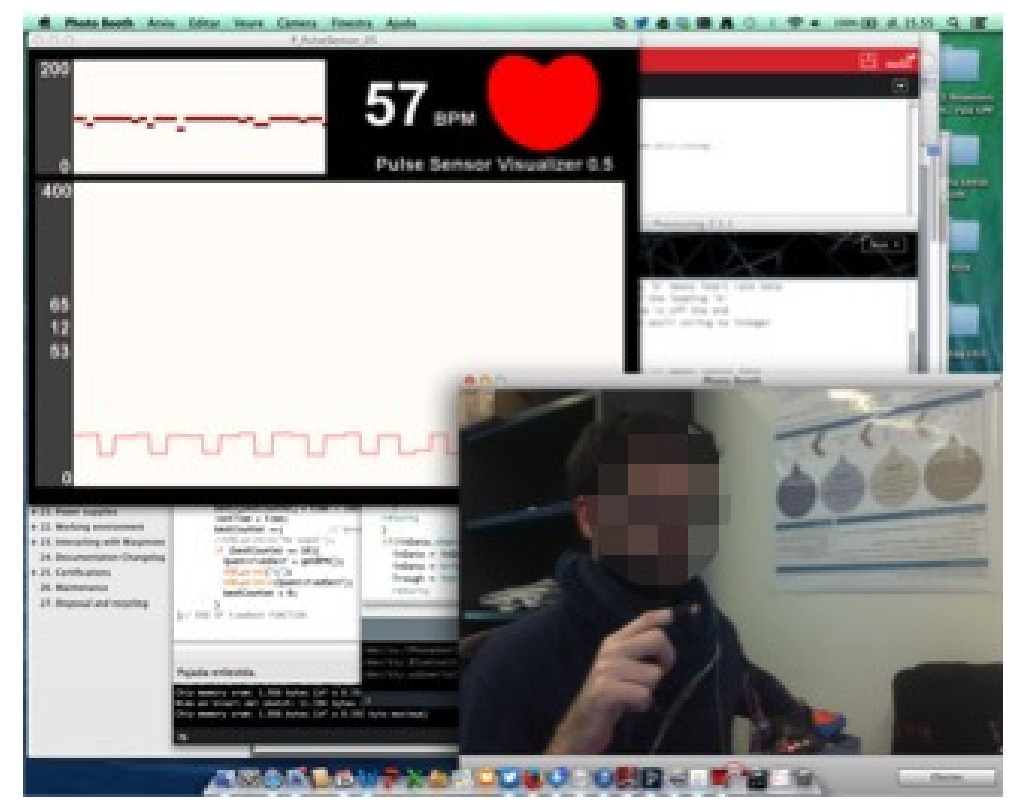

Figure 9: Measurement of the patient pulse using the CUIDATS wristband

- Reducing the considered number of measurement peaks to compute the BPM value would require less time of observation and processing, and therefore less energy consumption.

- To permanently track the patient, a constant estimation of its position is required. Assuming that a person walks at a velocity of $2 \mathrm{~m} / \mathrm{s}$, being in a sleeping state for more than 3 seconds could mean movements of more than 6 meters. To provide an accurate and energy-efficient tracking mechanism, the accelerometer placed in the wristband could send the wristband radio module to sleep if no movement was detected and there was not any BPM computation in progress.

\subsubsection{Escape alert}

The previously detailed RTLS system provides room-level location but it is not precise enough to detect people exiting a hospital, which can be of utmost importance in case of patients with mental deficiencies. Furthermore, for preventing the stealing of medical assets (such as wheelchairs or walkers) it is also important to real-time detect if an object is passing through an specific door. 


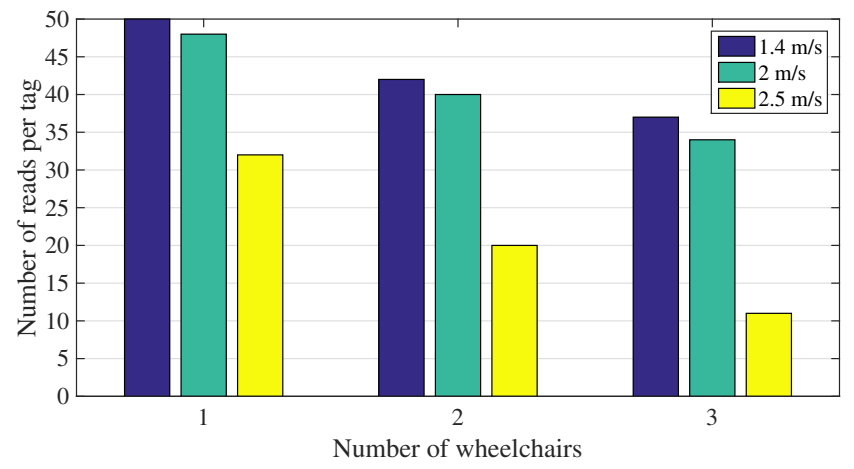

Figure 10: Number of readings per tag for detecting user escape

In order to evaluate the performance of our RFID system in these scenarios we placed an antenna (Keonn Advantenna-P11) in the ceiling of our laboratory and asked an user (carrying a passive UHF tag) to pass underneath at different speeds $(1.4,2$, and $2.5 \mathrm{~m} / \mathrm{s})$. Then, we asked a second user to do the same together with the first user, and finally we asked a third user to join the other two.

As expected, all tags were correctly detected in all situations. Then we repeated the same tests with the users sitting on (metallic) wheelchairs. Once again, all the tags were always detected, thus ensuring room-level accuracy. Figure 10 shows the mean number of readings per tag (with a read time of $200 \mathrm{~ms}$ ) considering one, two and three users, and three different speeds. Even in the worst case, when the three wheelchairs passed through the door at $2.5 \mathrm{~m} / \mathrm{s}$, each tag was read on average ten times, thus guaranteeing $100 \%$ of detection success.

\subsubsection{Asset inventory}

Although UHF RFID is a well-established technology for inventorying all types of objects, we performed specific tests for our application scenario. First, the metallic nature of wheelchairs and walkers could substantially decrease the reading capacities of RFID antennas (and readers) specified by Keonn Technologies. And second, in order to minimize the installation of antennas in the application scenario, it was important to characterize the reading capacity of our system.

For that, we placed the Keonn Advantenna-P11 in one of the walls of our laboratory and conducted several tests putting 20 metallic objects (with the 


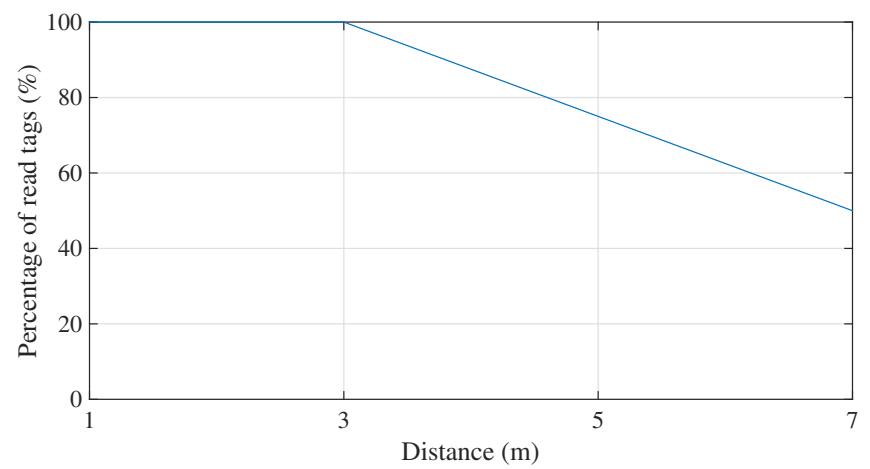

Figure 11: Percentage of RFID readings in function of the distance to the tag

corresponding RFID tag attached) at different distances from the antenna. Figure 11 shows how all tags are always read up to 3 meters of distance, but further than that, the reading capacity (expressed in \% of read tags) of the RFID system is linearly decreasing. At 7 meters of distance only $50 \%$ of the tags were detected. From these results we could conclude that for the rehabilitation warehouse (Figure 12 (c) in red) only one antenna would be required for inventorying all the material while guaranteeing room-level accuracy. Likewise, more antennas would be necessary to ensure bed-level accuracy to locate assets in room sub-locations.

Regarding the RFID tags with sensors [57, 59, 60], based on some initial tests in the laboratory, their reduced reading range discarded their utilization in the final version of the platform. However, this read range is expected to be increased in the near future, thanks to the constant improvement and miniaturization of the electronic components. Hence, we left the platform compatible with the RFID tags with sensors, to include them as soon as larger reading distances are available. Nevertheless, the vital signs monitoring capabilities are fully covered with the remaining wristband components.

\section{Pilot}

An important concern when dealing with technology and health care institutions is the usefulness, and even more important, the degree of acceptance of a new technology by the staff. In order to validate the CUIDATS system in a real health care environment, different tests were carried out in the facilities of the Hospital Asepeyo Sant Cugat del Vallès (Barcelona) with the 
collaboration of nursing, surgery, and rehabilitation departments.

We organized several meetings with the Hospital Board of Directors prior to the pilot deployment that helped us to plan tests in detail. For example, the floor maps of the three departments involved in the pilot (Figure 12) were essential for evaluating our technologies in the most realistic testbed as well as for identifying the number of required devices and designing their best distribution within the hospital. And more importantly, we had to face a trade-off between minimizing the impact of our pilot in the everyday activities of Asepeyo and maximizing the validation of the full potential of our system. Finally, we agreed to deploy the pilot during two days and evaluate the following use cases:

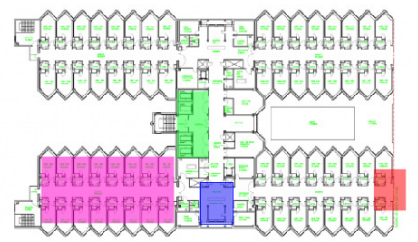

(a) Hospitalization floor

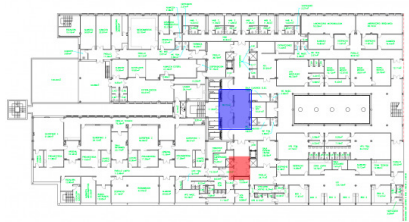

(b) Surgery floor

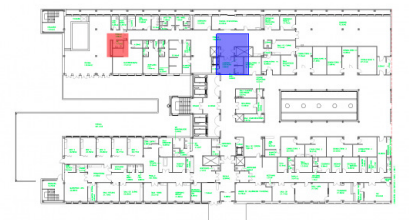

(c) Rehabilitation floor

Figure 12: Pilot scenario floor maps where application areas have been highlighted

\subsection{Use cases description}

1. Patient tracking and monitoring in the hospitalization floor When a patient registers in the hospitalization floor (Figure 13 (a)), they receive a wristband that will localize them, monitor their temperature and pulse and detect possible falls. In our pilot, we first tested how the system accurately localized the patient and activated an alarm if they approached the emergency door, and second, how the system activated an alarm if a patient fall was detected.

2. Patient tracking and monitoring before accessing the surgery room The time just before and just after a surgery is critical for any patient. For this reason, the CUIDATS wristband should be kept by the patient until entering the surgery room (Figure $13(\mathrm{~b})$ ), where other monitoring systems are already available. In our pilot we tested how the system monitored patient's vital signs and activated an alarm if their temperature over-passed 37 degrees when being near the operating room. 


\begin{tabular}{|c|c|c|c|c|c|c|}
\hline Use case & Wristbands & RFID tags & WSN Beacons & RFID Ant. & Users & Area covered \\
\hline $\mathbf{\# 1}$ & 1 & 0 & 4 & 0 & 5 & $507,29 \mathrm{~m}^{2}$ \\
\hline $\mathbf{\# 2}$ & 1 & 3 & 2 & 2 & 3 & $86,72 \mathrm{~m}^{2}$ \\
\hline $\mathbf{\# 3}$ & 0 & 10 & 3 & 2 & 5 & $57.7 \mathrm{~m}^{2}$ \\
\hline
\end{tabular}

Table 6: Elements employed during each pilot use case

3. Asset tracking near the rehabilitation department warehouse The rehabilitation department of the hospital (Figure 13 (c)) was having issues when inventorying the wheelchairs of its area. Although there is a special procedure for accompanying patients to the hospital exit, they often take the initiative of going out by themselves which leads to the lost (and sometimes even stealing) of wheelchairs. In our pilot we tested first, that all the wheelchairs available in the warehouse were localized by the system at any time, and second, how the system activated an alarm if a wheelchair abandoned the rehabilitation area.

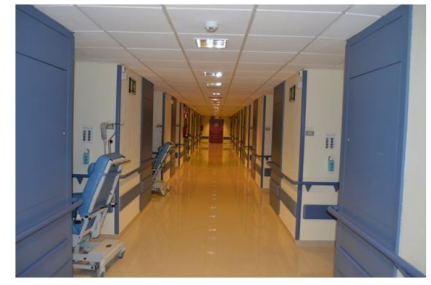

(a) Hospitalization area

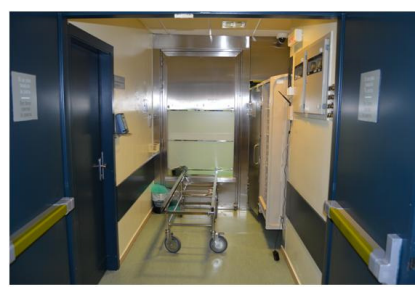

(b) Surgery area

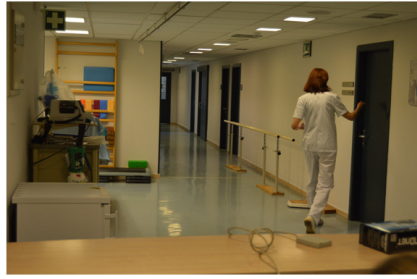

(c) Rehabilitation area

Figure 13: Images of the pilot application areas in the Asepeyo hospital

Table 6 details the amount of hardware equipment, number of users involved and covered area (in square meters) for the pilot of each use case.

\subsection{Evaluation of the pilot}

A thorough evaluation of our system in a testbed prior to the pilot, together with a good coordination with the pilot facilities management (Asepeyo) since the early start of the project, allowed a successful evaluation of the identified use cases.

The evaluation of the CUIDATS system at the Asepeyo hospital was meant to validate in a qualitative way the results obtained in the testbed previously described. Our intention was therefore to obtain a positive impression of the different use cases described in subsection 5.1 from the hospital staff. 
In that sense, two types of surveys were distributed among the hospital staff involved in the pilot: one for the management staff and another for the health care workers. The former were mainly asked about a future integration of the CUIDATS system with the existing HIS, about the value of the information supplied by the CUIDATS system for taking management decisions, and finally, if they would like to buy a monitoring system. The latter were asked mainly about the usability of the system and the activities in which they would appreciate the support of a platform like CUIDATS. In total we received feedback from 22 individuals, and the main conclusions are the following:

- The CUIDATS system can be easily integrated with the Asepeyo HIS platform and the installation of all the required devices (mainly antennas) would not be intrusive.

- The monitoring and tracking of patients and assets would be very useful in order to optimize working procedures defined from the management department.

- They do not think of buying a system like CUIDATS at present because they see it as a pre-industrial product and there are many European regulations that would need to be satisfied in order to be deployed in a real hospital.

- Health care workers identified the tracking of assets (Figure 14 (a)) and the supervision of patients' rehabilitation exercises as the two most common activities that make them lose a lot of time. So they would be very happy (and more efficient in other tasks) if they received the CUIDATS support for these activities.

- The CUIDATS application is intuitive and friendly. The visualization of a 3D-model of the hospital was especially appreciated. (Figure 14 (b))

\section{Conclusion and Future Work}

High-quality patient care and asset management have always relied on trustworthy information. In a non-far future, this information will increasingly come from smart health applications and be used for effective communication, collaboration, monitoring, and decision making in the context 


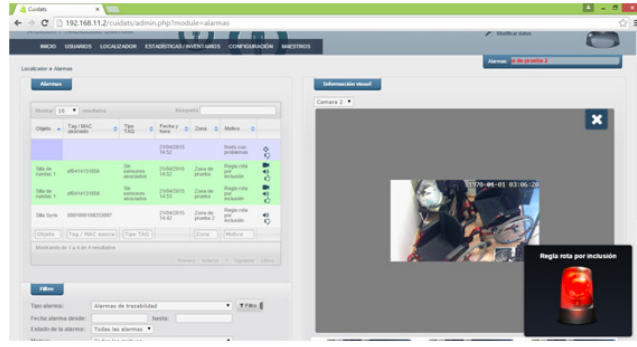

(a) New alarm detected

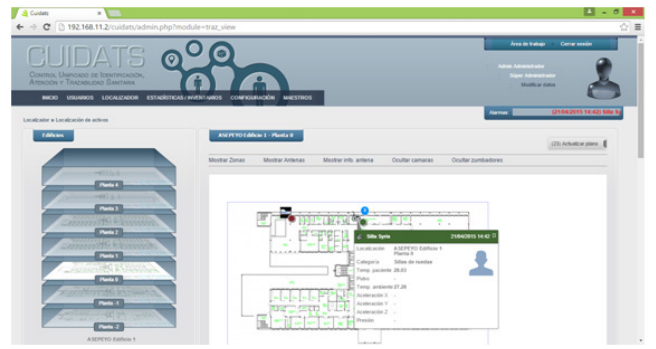

(b) Patient identification and location

Figure 14: CUIDATS dashboard screenshots

of Smart Cities. CUIDATS tries to solve some shortcomings of well-known wireless technologies when running patient and/or asset end-to-end monitoring systems in health care environments. RFID and WSN have been therefore successfully combined by creating a single hybrid network.

The final adoption of the CUIDATS approach in the evolving application field of smart health requires more research efforts, particularly in the hardware side. RFID tags have proven to successfully identify moving assets, however, the RFID tags with sensors are not yet capable to operate at a reasonable distance for patient monitoring. As for the WSN, it would be highly recommendable to use a sizing software to estimate the optimum number and position of beacons in any application scenario. In addition, alternatives in low-powered communication radio modules should be also considered for improving the patient data flow and the accuracy of the localization system. The integration of all the electronic elements in a smaller device is a challenge both in the wristband and in the WSN gateway, the former also needing a more powerful battery and the latter higher memory capabilities.

Different functionalities of the platform have been verified in a testbed, such as the locating system, the patient vital signs monitoring, the patient fall and escape alert systems, and the asset inventory system. From the evaluation of our developed pilot, we can conclude that the CUIDATS platform guarantees the required transparency of the system thanks to an optimal integration of different IoT technologies, also adding value for the health care staff as they have actively contributed in its design, implementation, and evaluation. Specifically, we achieved the following goals within the CUIDATS project:

- A transparent, flexible, low-cost, low-powered, and minimally interfer- 
ing RFID-WSN hybrid network.

- A real-time tracking system able to obtain patient and asset location with zone- and room-level accuracy, respectively.

- A monitoring system based on an electronic wristband that reports data and activates alarms from pulse, temperature and movement of the patient.

- The validation of the whole system both in quantitative and qualitative terms after having analyzed its performance in a testbed and in a real health care scenario.

Future work involves addressing the current hardware limitations described above, focusing on the RFID sensing capabilities, efficiency of the radio transmission in the WSN network, and wristband integration.

\section{Acknowledgments}

This work was partially supported by the Spanish Government through the projects TEC2012-32354, IPT-2012-0972-300000, and TEC2015-71303-

$\mathrm{R}$, and by the Catalan Government (SGR2009\#00617). The authors also acknowledge the rest of members of the CUIDATS consortium (Proengsoft, Pulso Ediciones, and i2cat), the Asepeyo hospital, and the technical support received by Keonn Technologies and Farsens.

\section{References}

[1] "Sustainable Development Challenges", Department of Economic and Social Affairs, United Nations, Available at: https:// sustainabledevelopment.un.org/, Accessed: 2015-10-11.

[2] A. Solanas, C. Patsakis, M. Conti, I. Vlachos, V. Ramos, F. Falcone, O. Postolache, P. Perez-Martinez, R. Pietro, D. Perrea, A. MartinezBalleste, Smart health: A context-aware health paradigm within smart cities, Communications Magazine, IEEE 52 (8) (2014) 74-81. doi:10. 1109/MCOM. 2014.6871673.

[3] EPCglobal, EPC Radio-Frequency Identity Protocols Generation-2 UHF RFID, Specification for RFID Air Interface, Protocol for Communications at $860 \mathrm{MHz}-960 \mathrm{MHz}$, Version 2.0.0 Ratified (2013). 
[4] Global Industry Analysts, Inc., Real-time location system (RTLS) solutions - A global strategic business report, http://www.strategyr.com/Real_Time_Location_System_RTLS_ Solutions_Market_Report.aspl, Accessed: 2015-10-05 (February 2013).

[5] ReportsnReports, RTLS in Healthcare Market - Analysis and Forecast to 2014 - 2020, http://www.reportsnreports.com/reports/ 274311-real-time-location-systems-rtls-market-by-product-tags-sensors-softwar html, Accessed: 2015-08-28 (July 2014).

[6] S. Gezici, A survey on wireless position estimation, Wireless personal communications 44 (3) (2008) 263-282.

[7] H. Liu, M. Bolic, A. Nayak, I. Stojmenović, Taxonomy and challenges of the integration of RFID and wireless sensor networks, Network, IEEE 22 (6) (2008) 26-35.

[8] J. Mitsugi, T. Inaba, B. Pátkai, L. Theodorou, J. Sung, T. S. López, D. Kim, D. McFarlane, H. Hada, Y. Kawakita, et al., Architecture development for sensor integration in the EPCglobal network, Auto-ID Labs White Paper Series.

[9] D. Ranasinghe, K. Leong, M. Ng, D. Engels, P. Cole, A distributed architecture for a ubiquitous RFID sensing network, in: Intelligent Sensors, Sensor Networks and Information Processing Conference, 2005. Proceedings of the 2005 International Conference on, IEEE, 2005, pp. $7-12$.

[10] P. G. Ansola, A. García, J. de las Morenas, J. G. Escribano, F. J. Otamendi, ZigID: Improving visibility in industrial environments by combining WSN and RFID, Journal of Zhejiang University SCIENCE A 12 (11) (2011) 849-859.

[11] L. Zhang, Z. Wang, Integration of RFID into wireless sensor networks: architectures, opportunities and challenging problems, in: Grid and Cooperative Computing Workshops, 2006. GCCW'06. Fifth International Conference on, IEEE, 2006, pp. 463-469. 
[12] E. Lai, A. Redfern, P. Wright, Vibration powered battery-assisted passive RFID tag, in: Embedded and Ubiquitous Computing-EUC 2005 Workshops, Springer, 2005, pp. 1058-1068.

[13] T. S. López, D. Kim, G. H. Canepa, K. Koumadi, Integrating wireless sensors and RFID tags into energy-efficient and dynamic context networks, The Computer Journal 52 (2) (2009) 240-267.

[14] N. Cho, S.-J. Song, S. Kim, S. Kim, H.-J. Yoo, A 5.1- $\mu$ W UHF RFID tag chip integrated with sensors for wireless environmental monitoring, in: Solid-State Circuits Conference, 2005. ESSCIRC 2005. Proceedings of the 31st European, IEEE, 2005, pp. 279-282.

[15] H. Kitayoshi, K. Sawaya, Long range passive RFID-tag for sensor networks, in: IEEE Vehicular Technology Conference, Vol. 62, IEEE; 1999, 2005, p. 2696.

[16] M. Philipose, J. R. Smith, B. Jiang, A. Mamishev, S. Roy, K. SundaraRajan, Battery-free wireless identification and sensing, Pervasive Computing, IEEE 4 (1) (2005) 37-45.

[17] A. Nedelcu, V. Stoianovici, I. Szekely, Energy-Efficient Integration of WSNs with Active RFID Systems, Bulletin of the "Transilvania" University of Braşov, Series I, Engineering Sciences 53 (2011) 127-135.

[18] H. Yang, F. Yao, S.-H. Yang, Zigbee enabled radio frequency identification system.

[19] H. Ramamurthy, B. Prabhu, R. Gadh, A. M. Madni, Wireless industrial monitoring and control using a smart sensor platform, Sensors Journal, IEEE 7 (5) (2007) 611-618.

[20] C. Englund, H. Wallin, RFID in wireless sensor network, CU of Technology, editor, Master's Thesis, Gotenborg, Sweden.

[21] M. P. Lewis, K. W. Hsu, TelosRFID: An ad-hoc wireless networking capable multi-protocol RFID reader system, in: Radio and Wireless Symposium (RWS), 2010 IEEE, IEEE, 2010, pp. 392-395.

[22] W. Brunette, J. Lester, A. Rea, G. Borriello, Some sensor network elements for ubiquitous computing, in: Information Processing in Sensor 
Networks, 2005. IPSN 2005. Fourth International Symposium on, IEEE, 2005, pp. 388-392.

[23] B. Torres, Q. Pang, G. Skelton, S. Bridges, N. Meghanathan, Integration of an RFID Reader to a Wireless Sensor Network and using it to Identify an Individual Carrying RFID Tags, arXiv preprint arXiv:1105.0066.

[24] M. J. Franklin, S. R. Jeffery, S. Krishnamurthy, F. Reiss, S. Rizvi, E. Wu, O. Cooper, A. Edakkunni, W. Hong, Design Considerations for High Fan-In Systems: The HiFi Approach, in: CIDR, 2005, pp. 290-304.

[25] K. Emery, Distributed eventing architecture: RFID and sensors in a supply chain.

[26] J. Sung, T. S. Lopez, D. Kim, The EPC sensor network for RFID and WSN integration infrastructure, in: Pervasive Computing and Communications Workshops, 2007. PerCom Workshops' 07. Fifth Annual IEEE International Conference on, IEEE, 2007, pp. 618-621.

[27] P. Fuhrer, D. Guinard, Building a Smart Hospital using RFID Technologies, ECEH 91 (2006) 131-142.

[28] G. Virone, A. Wood, L. Selavo, Q. Cao, L. Fang, T. Doan, Z. He, J. Stankovic, An advanced wireless sensor network for health monitoring, in: Transdisciplinary Conference on Distributed Diagnosis and Home Healthcare (D2H2), 2006, pp. 2-4.

[29] Y. Zhang, L. T. Yang, J. Chen, RFID and sensor networks: architectures, protocols, security, and integrations, CRC Press, 2009.

[30] R. Parada, J. Melia-Segui, M. Morenza-Cinos, A. Carreras, R. Pous, Using RFID to detect interactions in ambient assisted living environments, Intelligent Systems, IEEE 30 (4) (2015) 16-22. doi:10.1109/ MIS . 2015.43.

[31] L. Ho, M. Moh, Z. Walker, T. Hamada, C.-F. Su, A prototype on RFID and sensor networks for elder healthcare: progress report, in: Proceedings of the 2005 ACM SIGCOMM workshop on Experimental approaches to wireless network design and analysis, ACM, 2005, pp. 70-75. 
[32] H. Deng, et al., Design of sensor-embedded radio frequency identification (SE-RFID) systems, in: 2006 International Conference on Mechatronics and Automation, 2006, pp. 792-796.

[33] A. Wickramasinghe, D. Ranasinghe, Ambulatory monitoring using passive computational RFID sensors, Sensors Journal, IEEE PP (99) (2015) 1-1. doi:10.1109/JSEN.2015.2449862.

[34] S.-J. Kim, S. K. Yoo, H.-O. Kim, H.-S. Bae, J.-J. Park, K.-J. Seo, B.-C. Chang, Smart blood bag management system in a hospital environment, in: Personal wireless communications, Springer, 2006, pp. 506-517.

[35] M.-S. Jang, M.-S. Goh, E.-H. Lee, S.-B. Choi, Design and Implementation of a Wristband-style Biomedical Signal Measurement Device using Zigbee for u-Healthcare Systems, Int. Journal of Computer Science and Network Security 11 (3) (2011) 47-54.

[36] M. Chan, D. Estève, J.-Y. Fourniols, C. Escriba, E. Campo, Smart wearable systems: Current status and future challenges, Artificial intelligence in medicine 56 (3) (2012) 137-156.

[37] G. Appelboom, E. Camacho, M. E. Abraham, S. S. Bruce, E. L. Dumont, B. E. Zacharia, R. D'Amico, J. Slomian, J. Y. Reginster, O. Bruyère, et al., Smart wearable body sensors for patient self-assessment and monitoring, Archives of Public Health 72 (1) (2014) 1.

[38] S. Patel, H. Park, P. Bonato, L. Chan, M. Rodgers, A review of wearable sensors and systems with application in rehabilitation, Journal of neuroengineering and rehabilitation 9 (1) (2012) 1.

[39] A. Pantelopoulos, N. G. Bourbakis, A survey on wearable sensor-based systems for health monitoring and prognosis, IEEE Transactions on Systems, Man, and Cybernetics, Part C (Applications and Reviews) 40 (1) (2010) 1-12.

[40] L. Gatzoulis, I. Iakovidis, Wearable and portable ehealth systems, IEEE Engineering in Medicine and Biology Magazine 26 (5) (2007) 51-56.

[41] T. Yilmaz, R. Foster, Y. Hao, Detecting vital signs with wearable wireless sensors, Sensors 10 (12) (2010) 10837-10862. 
[42] S. Weber, P. Scharfschwerdt, T. Schauer, T. Seel, U. Kertzscher, K. Affeld, Continuous wrist blood pressure measurement with ultrasound, Biomedical Engineering/Biomedizinische Technik.

[43] Y. Khan, A. E. Ostfeld, C. M. Lochner, A. Pierre, A. C. Arias, Monitoring of vital signs with flexible and wearable medical devices, Advanced Materials.

[44] S. Perkins, New wristband measures sweat to monitor health risks, http://www.sciencemag.org/news/2016/01/ new-wristband-measures-sweat-monitor-health-risks, Accessed: 2016-10-16. doi:10.1126/science.aae0282.

[45] J. Dusheck, Wearable device detects, analyzes realtime changes in chemical composition of sweat, https://med.stanford.edu/news/all-news/2016/01/ wearable-device-detects-real-time-changes-in-composition-of-sweat . html, Accessed: 2016-10-16.

[46] Awarepoint website, http://www.awarepoint.com/, Accessed: 2015$10-11$.

[47] Centrak website, http://www. centrak.com/, Accessed: 2015-10-11.

[48] Ekahau website, http://www.ekahau.com/, Accessed: 2015-10-11.

[49] Radianse website, http://www.radianse.com/, Accessed: 2015-10-11.

[50] Sonitor website, http://www. sonitor.com/, Accessed: 2015-10-11.

[51] Stanley Healthcare website, http://www.stanleyhealthcare.com/, Accessed: 2015-10-11.

[52] Zebra website, https://www.zebra.com, Accessed: 2015-10-11.

[53] PointRF website, http://www.pointrf .com/, Accessed: 2015-10-11.

[54] Teletracking website, http://www.teletracking.com/, Accessed: 2015-10-11.

[55] Versus Technology website, http://www.versustech.com/, Accessed: 2015-10-11. 
[56] RFID Global Solution website, http://www.rfidgs.com/, Accessed: 2015-10-11.

[57] I. Zalbide, E. D'Entremont, A. Jimenez, H. Solar, A. Beriain, R. Berenguer, Battery-free wireless sensors for industrial applications based on UHF RFID technology, in: Sensors, 2014 IEEE, 2014, pp. 1499-1502. doi:10.1109/ICSENS. 2014.6985299.

[58] ShortDipole Impinj Monza 5 RFID tag, http://www. smartrac-group. com/, Accessed: 2015-10-13.

[59] Farsens: EPC C1G2 compliant batteryless orientation sensor: KINEOA3DH, http://www.farsens.com/en/magnitudes/orientation, Accessed: 2015-10-13.

[60] Farsens: EPC C1G2 compliant batteryless temperature sensor: PYROS0373, http://www.farsens.com/en/magnitudes/temperature, Accessed: 2015-10-13.

[61] Keonn Technologies, RFID UHF AdvanReader-150 Datasheet, http: //keonn.com/images/stories/pdf/Keonn-AdvanReader-150-Data_ sheet.pdf, Accessed: 2015-09-03.

[62] Libelium, Waspmote Datasheet, http://www.libelium.com/ v11-files/documentation/waspmote/waspmote-datasheet_eng. pdf, Accessed: 2015-09-03.

[63] Keonn Technologies, RFID Advantenna-p11 Datasheet, http://keonn. com/images/stories/pdf/Keonn-Advantenna-p11-Data_sheet.pdf, Accessed: 2015-09-03.

[64] Beagleboard.org, BeagleBone Black Datasheet, http://beagleboard. org/black, Accessed: 2015-09-03.

[65] N. Bulusu, J. Heidemann, D. Estrin, GPS-less Low-cost Outdoor Localization for Very Small Devices, Personal Communications, IEEE 7 (5) (2000) 28 -34. doi:10.1109/98.878533.

[66] J. Blumenthal, R. Grossmann, F. Golatowski, D. Timmermann, Weighted Centroid Localization in Zigbee-based Sensor Networks, in: in IEEE International Symposium on Intelligent Signal Processing, WISP 2007, 2007, pp. 1-6. 